Leistungen von Agrarbetrieben richtig bewerten

\section{Was leistet die Landwirtschaft wirklich?}

\author{
Grundlage aller unternehmerischen Entscheidungen ist die betrieb- \\ liche Rechnungslegung, die die Wirtschaftskraft eines Betriebes \\ abbildet. Problematisch hierbei ist, dass in der traditionellen Rech- \\ nungslegung Natur- und Sozialkapital nicht berücksichtigt werden. \\ Dies gilt insbesondere für die Landwirtschaft. \\ Von Christian Hiß
}

D ie landwirtschaftliche Produktion wird von besonders vielen positiven und negativen Wirkungen und Effekten auf die Natur, die Umwelt, die Region, die Landschaft und die Gesellschaft begleitet. In den vergangenen Jahrzehnten wurde ihnen in der praktischen und theoretischen Betriebswirtschaft zu wenig Beachtung geschenkt. Der Fokus lag auf einem aus dem industriellen Wirtschaftsbegriff abgeleiteten Effizienzgebot, das die Steigerung des Ertrags pro Flächeneinheit einer Kulturpflanze oder pro Nutztier als Hauptparadigma verfolgte.

Tatsächlich konnten so mit immer weniger menschlicher Arbeitskraft gewaltige Mengen an Nahrungsmitteln erzeugt und in den Markt gebracht werden. Heute müssen wir feststellen, dass durch diesen Wirtschaftstyp aber auch Schäden, Verluste und Risiken an der Artenvielfalt, der Bodenfruchtbarkeit und dem Erdklima von gewaltigem Ausmaß entstanden sind. In der Folge des industriellen Paradigmas in der Betriebsführung sind Schäden an der natürlichen Grundlage und der gesellschaftlichen Verfassung entstanden.

\section{Unvollständige Rechnungslegung}

In der ökonomischen Theorie gelten bisher solche schädlichen Effekte als Externalitäten, weil sie in der privaten Rechnung eines Betriebes nicht zur Geltung kommen. Das ändert sich, denn gegenwärtig erleben wir einen generel- len Paradigmenwechsel in der ökonomischen Einordnung ökologischer und sozialer Faktoren des Wirtschaftens. Der Dürresommer 2018 hat offenbart, dass die in der Vergangenheit von der Art des Wirtschaftens erzeugten Wirkungen an den natürlichen Grundlagen als betriebliche Risiken und Verluste direkt wieder in die betriebswirtschaftliche Rechnung zurückkehren und nicht nur als fiktive oder reale volkswirtschaftliche Externalisierungskosten vorhanden sind.

\section{Vielfältige und spezialisierte Betriebe}

Viele landwirtschaftliche Betriebe hatten enorme Ertragseinbußen zu verzeichnen, die sich durch die wegen Verknappung erhöhten Preise nur teilweise wieder ausglichen. Der industriell spezialisierte Betrieb hatte keine Ausgleichsmöglichkeiten, der vielfältige Betrieb konnte dagegen besser kompensieren, weil nicht alle Kulturen gleichermaßen unter der Dürre litten. Einen ähnlichen Effekt kann man beim Rückgang der Insektenpopulationen beobachten. Kulturen, deren Erträge von der Bestäubung durch Insekten abhängig sind, erleiden Ertragseinbußen. Die zunächst externalisierten Kosten des Insektensterbens kehren wieder in den Betrieb zurück und machen sich dort finanziell bemerkbar.

Lange Zeit galten ökologisch und vielfältig arbeitende Betriebe als ideell bis ideologisch motiviert, den Nimbus des effektiven und fortschrittlichen Wirtschaftens hatten die industriell ar- beitenden Unternehmen für sich proklamiert. Es wird aber immer deutlicher, dass die ökologisch sorgsam arbeitenden Betriebe die besseren Unternehmer/innen im ökonomischen Sinne sind. Durch ihre Art des Wirtschaftens vermeiden sie Verluste, Schäden und Risiken an den natürlichen Vermögensanlagen ihres Betriebes, indem sie die ökologischen und sozialen Faktoren in ihren Betriebsablauf internalisieren und sich im Anbau breiter aufstellen. Vielfältiges Wirtschaften ist aufwendiger und teurer, das Umschlagen der höheren Kosten auf die Produktpreise ist dem ökologischen Betrieb in der Vergangenheit zwar teilweise gelungen, weil immer mehr Konsument/innen ihrer Rechenlogik folgen konnten, einen wirklichen Durchbruch konnte der ökologische Landbau bis zur Gegenwart aber nicht verzeichnen.

\section{„Richtig rechnen“ ist geboten}

Wie konnte es über Jahrzehnte passieren, dass sich ein falsches Wirtschaftsverständnis hartnäckig hält, obwohl offensichtlich war, dass die landwirtschaftliche Praxis in der Art langfristig nicht haltbar ist. Die Ursache dafür ist eine unvollständige betriebswirtschaftliche Rechenlogik. Das Instrument der Buchhaltung und Bilanzierung ist bis heute blind auf dem Auge des Natur- und Sozialkapitals, obwohl diese die existenziellen Grundlagen des Wirtschaftens darstellen (Hiß 2016).

Ein Beispiel verdeutlicht den Gedanken. Durch die Bewirtschaftung von Ackerboden wird Bodenfruchtbarkeit abgebaut, die Wiederherstellung durch die gezielte Zufuhr organischen kohlenstoffhaltigen Materials bedeutet betrieblichen Aufwand. Die dafür entstehenden Kosten werden zwar als Kosten verbucht, nicht aber dem Betriebsvermögen gutgeschrieben. Das müsste eigentlich geschehen, denn Bodenfruchtbarkeit ist ein Betriebsvermögen des landwirtschaftlichen Betriebes. Der Ersatz durch synthetische Dünger hat zwar die Produktion von Kulturpflanzen ermöglicht, nicht aber die organische Substanz 
erhalten. So fehlen dem Boden wesentliche Eigenschaften, wie die Wasserhaltekraft oder die Bindefähigkeit von $\mathrm{CO}_{2}$. Der Dürresommer hat gezeigt, dass mit Humus gut aufgebaute Böden die Ertragsfähigkeit unter Extrembedingungen besser ausgleichen konnten als Böden, die weniger Humus enthalten.

So wie das Beispiel zeigt, können viele soziale, ökologische und regionalwirtschaftliche Parameter als Betriebsvermögen eingeordnet werden. Deshalb ist es geboten, dass die Betriebe künftig auch diese Leistungen ihrer betrieblichen Tätigkeit erfassen, nachweisen und sie als Teil des betriebswirtschaftlichen Geschäftsergebnisses bewerten.

\section{Neue Instrumente und Methoden}

Um das Erfordernis umsetzen zu können, braucht es erweiterte Buchhaltungs- und Bilanzierungsinstrumente zur Erfassung und Methoden zur Bewertung der betrieblichen Leistungen zum Natur- und Sozialkapital (Hiß 2015). Die Grundlage dafür bilden die in der gewöhnlichen Finanzbuchhaltung bereits vorhandenen Daten und die durch den Betrieb zusätzlich erhobenen Informationen. Der methodische Ansatz liegt in der ,verursachungsgerechten Zuordnung von Aufwänden und Erträgen“ anhand von Belegen der Rechnungslegung und deren Verwertung.

Es soll mithilfe von Indikatoren und zusätzlichen Konten möglichst genau gezeigt werden, welche Kosten und welcher Mehraufwand im landwirtschaftlichen Betrieb für nachhaltiges Wirtschaften entstehen. Der Betrieb zeigt damit den geldwerten betrieblichen Aufwand, den er tatsächlich geleistet hat, damit die Bodenfruchtbarkeit erhalten oder der Lebensraum für einen Schmetterling geschaffen wird. Folglich wird nicht dem Schmetterling oder der Bodenfruchtbarkeit ein Wert zugeschrieben, sondern dem einzelnen Betrieb, der Maßnahmen ergreift oder nicht ergreift für die Erhaltung dessen Lebensraums und des Fruchtbarkeitspotenzials seiner landwirtschaftlich genutzten Flächen.
Die Methode umfasst vier aufeinanderfolgende Schritte:

- Als Erstes erfolgt die Erfassung der Nachhaltigkeitsleistungen durch den Betrieb anhand von Indikatoren und Erfassungskonten die im Regionalwert-Erfassungsinstrument vorgeschlagen werden. Eine wesentliche Quelle für die Daten und Informationen ist die Summen- und Saldenliste, in ihr sind viele Zahlen von Aufwänden und Erträgen versteckt, die für soziale und ökologische Wertaussagen gebraucht werden. Eine zweite Quelle von Informationen sind Zeiterfassungstabellen für die spezifischen Arbeiten, wie Kompostherstellung, Maßnahmen zur Erhöhung der Biodiversität oder Leistungen zur Ausbildung von jungen Menschen. Hier bietet das Regionalwert-Erfassungsinstrument schon eine Vielzahl von Indikatoren. Das Tool erlaubt dem Betrieb, weitere Indikatoren und Erfassungskonten einzufügen und einen eigenen Kontenplan zu erstellen. Auf dieser Stufe liegen die Informationen entweder bereits in Geldwerten vor, wenn zum Beispiel eine Rechnung für einen Einkauf dem neuen Konto zugeordnet werden kann. Es können auch andere Einheiten, wie Arbeitsstunden, Produktionsmengen, Flächenmaße oder Abschreibungszyklen vorgegeben sein.

- Ein Teil der in der Buchhaltung erfassten Daten und Informationen können anschließend direkt in die Berechnung übernommen, ein weiterer Teil muss einer Interpretation und Bewertung unterzogen werden, da einige Indikatoren für sich genommen noch keine bewertete Aussage treffen. Die Referenzwerte können je nach Betrieb, Region oder Branche variieren, sie sind aber transparent und nachvollziehbar. Zur Interpretation der vorliegenden betrieblichen Daten können Kennzahlen oder Sollzahlen verwendet werden. Am Ende der Stufe liegen auf jeden Fall monetäre Werte zur Weiterverarbeitung vor.

I Diese monetären Werte werden kumuliert oder einzeln dargestellt und ausgewiesen. Auf dieser Stufe ist es möglich, verschiedene Leistungsparameter in einen einzelnen zusammenzuziehen. Zum Beispiel werden die Leistungen zur Erhaltung und zum Aufbau von Bodenfruchtbarkeit in unterschiedlichen Konten erfasst. Der Anbau von Gründüngungspflanzen, die Herstellung und die Ausbringung von Kompost und die spezifische bodenschonende Bearbeitung des Bodens durch teure Spezialmaschinen und die Behandlung mit spezifischen Präparaten zählen dazu. Selbst die Teilnahme des Betriebsleitenden an Fortbildungsmaßnahmen zu Bodenfruchtbarkeit kann als Aufwand veranschlagt werden. Im Anschluss werden die verschiedenen Konten in das Vermögenskonto Bodenfruchtbarkeit zusammengezogen.

- Auf der vierten Stufe werden nun die monetären Werte aus der sozialen, ökologischen und regionalwirtschaftlichen Leistungserfassung in der Gewinn- und Verlustrechnung sachgerecht zugeordnet. Sie sind einzeln und summiert in einem erweiterten Jahresabschluss per Überleitung in eine Nachhaltigkeitsbilanz darstellbar und stehen unterschiedlichen Verwertungsarten zur Verfügung.

\section{Was hat der Betrieb davon?}

Warum ist die Überarbeitung der Finanzbuchhaltung und der Bilanzregeln so wichtig für die ökologisch-ökonomische Wende? Können die Betriebe nicht trotzdem ökologischer und sozialer wirtschaften, auch wenn die betriebswirtschaftlichen Instrumente unverändert bleiben?

Die Erfahrung zeigt, dass die ökologischen und sozialen Faktoren erst dann ihre adäquate Wertigkeit in der Betriebsführung erhalten, wenn sie sich in der abstrakten Rechnungslegung und monetär in der Kasse der Betriebe bemerkbar machen. Es muss sich nicht nur auf dem Acker, sondern auch in der abstrahierenden Bilanz zeigen, dass das Betriebskapital Bodenfruchtbarkeit in ei- 
nem Wirtschaftsjahr zugenommen oder abgenommen hat.

Für den Betrieb, der die RegionalwertNachhaltigkeitsbilanzierung erstellt, eröffnen sich diverse Möglichkeiten, mit den hinzugewonnenen Informationen umzugehen:

【 Sie können für die Unternehmenssteuerung und das Controlling verwendet werden,

I sie ermöglichen eine Bewertung von betriebswirtschaftlichen Risiken und Leistungen,

I sie sind Teil der Geschäftsberichterstattung an Kund/innen und Lieferanten,

- die finanziell nachgewiesenen Aufwände für Nachhaltigkeitsleistungen können in Rechnung gestellt werden, zum Beispiel an: Ausgleichs-Fonds, Mitglieder der Solidarischen Landwirtschaft, gemeinnützige Träger, CSRPartner, Eigentümer,

- bei den Produkt-Preiskalkulationen dienen sie als Datengrundlage,

- sie werden zur Festlegung von Markenkriterien und ihrer Kontrolle verwendet.

\section{Schnelle Effekte und langfristige Strategie}

Die am schnellsten wirksame Methode, Landwirtschaft nachhaltiger $\mathrm{zu}$ gestalten, ist die Bereitstellung von Ausgleichsfonds, aus denen die in der Bilanz der Betriebe nachgewiesenen Leistungen der Betriebe finanziell ausgeglichen werden können. Um beim Beispiel der $\mathrm{CO}_{2}$-Rückbindung zu bleiben, wäre das Verfahren so: Ein Unternehmen oder eine Privatperson haben ihre $\mathrm{CO}_{2}$-Emissionen bereits so weit reduziert, wie sie selbst dazu in der Lage sind. Den Rest bieten sie zur Kompensation durch die Landwirtschaft an. Der landwirtschaftliche Betrieb tritt mit seinem errechneten $\mathrm{CO}_{2}$-Rückbindungswert an den Markt heran und erhält dafür Geld für seinen betrieblichen Aufwand und die dadurch ausgelöste Wirkung. So wie bei der $\mathrm{CO}_{2}$-Rückbindungsleistung kann es auch für andere Leistungen, wie zum Beispiel Erhaltung und Schaffung von
Biodiversität und Bildung, bewerkstelligt werden. Geld für dieses Vorgehen wäre vorhanden, wenn die 55 Milliarden Euro die jedes Jahr an EU-Geldern für die Landwirtschaft ausgegeben werden, für eine solche leistungsbezogene Vergabe verwendet würden.

An die Wurzeln der vielfältigen Herausforderungen und zu einer langfristigen Veränderung des Wirtschaftens kommen wir aber erst, wenn die innere Informationsebene, sprich das gesamte betriebswirtschaftliche Instrumentarium, die Wirklichkeit der Unternehmen und ihres sozialen und ökologischen Umraums entsprechend der tatsächlichen Verhältnisse abbildet und wiedergibt. Hier könnte von lebenden Organismen gelernt werden. Ihre permanente epigenetische Interaktion zwischen Genotyp und Phänotyp muss Vorbild werden für den strukturellen Aufbau und für die Reaktionsmechanismen von Wirtschaftsunternehmen. In digitalen Zeiten dürfte die Verarbeitung der notwendigen gigantischen Informations- und Datenmengen auch nicht mehr das Problem sein.

\section{Literatur \\ Hiß, C. (2015): Richtig rechnen. Mit der Reform der Finanzbuchhaltung zur ökologisch- ökonomischen Wende. München, oekom. Hiß, C. (2016): Der Bauer ist kein Händler In: Scheidewege 46: 391-403.}

AUTOR + KONTAKT

Christian Hiß ist Gründer und Vorstand der Regionalwert AG Freiburg, er ist gelernter Gemüsebauer und hat einen Masterabschluss in Social Banking and Social Finance. Er entwickelt seit einigen Jahren die Regionalwert AC Bürgeraktiengesellschaft als neuartige Intermediärstruktur zur regionalen Landund Ernährungswirtschaft. Die Erweiterung der Buchhaltung und Bilanzierung zur zeitgemäßen Abstraktion von Unternehmenserfolg ist sein zweites Arbeits- und Forschungsgebiet.

Regionalwert AG Freiburg, Christian Hiß, Geyer-zu-Lauf-Straße 5, 79312 Emmendingen. Tel.: +49 7 6419592615, E-Mail: hiss@regionalwert-ag.de, Website: www.regionalwert-ag.de

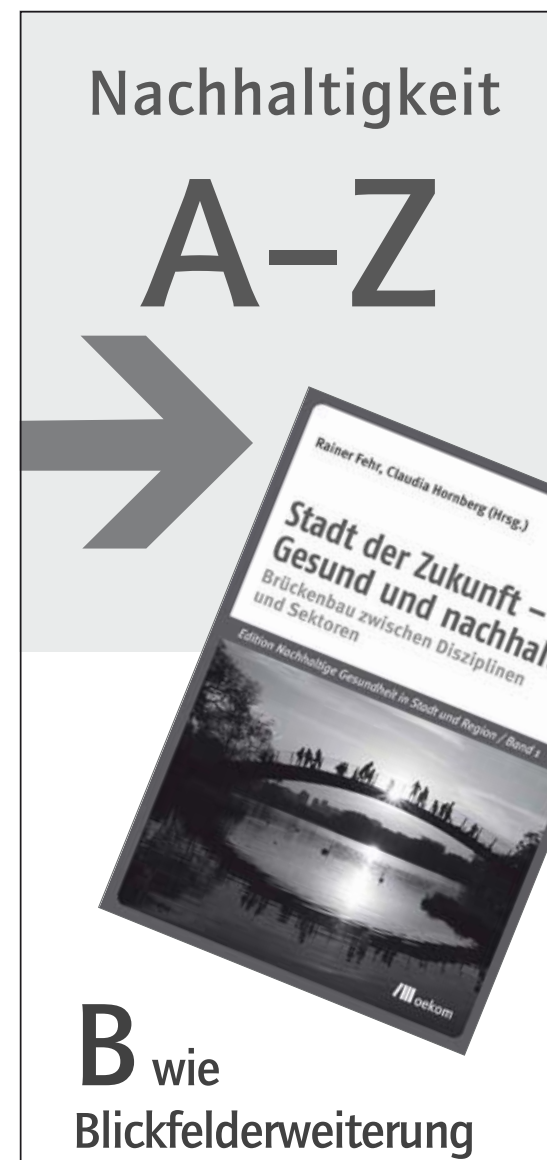

Am gesellschaftlichen Einsatz für Förderung. Schutz und Wiederherstellung von Gesundheit sind im städtischen Leben zahlreiche Akteure und Bereiche beteiligt. Während soziale Benachteiligung oft als wichtiger Faktor wahrgenommen wird, ist die Aufmerksamkeit für ökologische Aspekte bisher geringer. Nötig ist daher eine "Blickfelderweiterung" in Richtung Zukunftsvorsorge und Nachhaltigkeit. Dafür entwickeln die Autor(inn)en ein umfassendes Konzept Nachhaltiger StadtGesundheit als Erkenntnis- und Handlungsansatz.

R. Fehr, C. Hornberg (Hrsg.)

Stadt der Zukunft - Gesund und nachhaltig

Brückenbau zwischen Disziplinen und Sektoren

384 Seiten, broschiert, 35,- Euro ISBN 978-3-96238-074-8

Erhältlich im Buchhandel oder versandkostenfrei innerhalb Deutschlands bestellbar unter www.oekom.de. Auch als E-Book erhältlich.

\section{/II oekom}

Die guten Seiten der Zukunft 